\title{
Rates of Allocation for Disability Items by Mode in the American Community Survey
}

\author{
Carlos Siordia \\ Department of Epidemiology, GSPH, University of Pittsburgh \\ 130 North Bellefield Ave, Pittsburgh, PA 15213, USA \\ E-mail: csiordia@gmail.com
}

Received: March 2, 2015 Accepted: March 20, 2015 Published: March 26, 2015

doi:10.5296/iss.v3i1.7178 URL: http://dx.doi.org/10.5296/iss.v3i1.7178

This work was supported by grant number T32 AG000181 and U01 AG023744 from the National Institute of Aging at the National Institutes of Health (to AB Newman).

\begin{abstract}
Knowing the prevalence of disability in a population is seen as informative to policy and public health efforts. The American Community Survey (ACS), a yearly, large scale population-based survey administered by the United States (US) Census Bureau, is the predominant source for estimating disability prevalence in the US population. To provide complete data, procedures such as "allocations" are used by the bureau to fix illogical and missing responses to survey questions. This study investigates the rate of allocation in disability items by using the ACS Public Use Microdata Sample (PUMS) 3-year (2009-2011) file. Rates of allocation are interpreted as a measure of "within-person item-completeness" (WIPC) - where the presences of high allocation rates signal low levels of questionnaire completeness. The highest rates of allocations were in found in mail mode for: males (3.5\%); those with no college (4.4\%); households who only speak Spanish (5.9\%); non-married family households (4.3\%); and for Non-Latino-Blacks (6.5\%). Researchers should continue to explore how the equilibrium of population estimates from ACS sample data vary as a function of demo- and geo-graphic characteristics.
\end{abstract}

Keywords: disability, public health, allocation, sex, race, ACS, PUMS 


\section{Introduction}

The use of surveys to study the human population has been around for many of years. Some report the earliest record of a mailed survey can be traced to 1788 and that a face-to-face scientific survey can be identified in 1912 (De Heer et al, 1999). The main reasons why individuals participate with surveys is a sense of social obligation, financial compensation, or the topic of research is of interest (Tourangeau, 2004). Survey data is popular as they are useful when attempting to infer characteristics of a population (i.e., universe) by using "estimates" (i.e., empirically derived approximations) from a randomly selected subset of their group (i.e., sample) (see Siordia, 2013).

A population estimate is derived from a sample to characterize the population from which it was drawn. For example, estimating disability prevalence in the United States (US) population (of more than 300 million people) could be done by only using information on 3 million randomly selected individuals. Producing population estimates from samples requires the use of complete datasets - where missing, illogical, or ambiguous responses are "edited" (i.e., allocated) with plausible values in data processing protocols.

The specific aim of this report is to show how rates of allocation vary by mode of data collection as a function of: sex; educational attainment; language spoken at home; marital status; and race-ethnicity. The project hopes to contribute to survey methodology research interested in estimating disability prevalence by showing how technical details in the production of complete datasets may influence the precision of prevalence estimates and their confidence intervals.

\subsection{Mode of Data Collection}

The idea of "modes" dates back many decades (Couper, 2011) when, for example, the differences between mail and direct interview were described as using "different kinds of canvass" (Deming, 1944) or as "strategies" elsewhere (Hochstim, 1967). Abstracting reality into the world of numbers via questionnaires can be influenced by various factors including the method (i.e., mode) used to collect the information. For example, the "locus of control" may be highest when participating with a survey through mail than when the study subject is interviewed by a person (where loci lies with interviewer) or in computer-assisted surveys (where loci lies with software) (Couper, 2011).

In the American Community Survey (ACS - described below), mail questionnaires are keyed after being checked-in and are then forwarded for a telephone follow-up (at centralized phone centers) if critical information is missing or several inconsistencies are noted (McGovern \& Griffin, 2003). If a household does not respond by mail or phone then an in-person interview is attempted. In this paper, responding by mail is simply referred to as MAIL mode (others refer to as PAPI-paper and pencil interviewing), responding by telephone as CATI (computer assisted telephone interviewing) and in-person response as CAPI (computer assisted personal interviewing) - these acronyms follow those used to explain mode of data collection for the ACS (see Fuchs et al, 2000; and Eaton et al, 2010). 
A discussion on mode of data collection is undertaken in this paper under the assumptions that non-ignorable differences in data quality exist between modes and that mode of participation does not occur at random. With regards to the latter, research has shown that: linguistically isolated households have lower percentages of response via MAIL mode (McGovern, 2004); married-households have better MAIL participation (Joshipura et al., 2008); language issues arise with CAPI in the ACS (Carrasco, 2003); and response rate differs by the use of multilingual solicitation brochures (Joshipura et al., 2010). Examining data by mode matters because allocation rates differ by the method used to collect information - where MAIL generally contains higher allocation rates as interviewers in CATI and CAPI mode may help clarify questions that would have otherwise been left unanswered.

Allocating (i.e. assigning) responses to missing or illogical survey items can be argued to be related to "measurement error" (flawed assessment of a phenomenon). For example, when a person is asked if they have 'difficulty with vision,' they may not respond to the question as they may be unsure if wearing glasses qualifies. Identifying this individual as 'visually disable' after his/her missing response is assigned an answer may have one of two types of measurement error: (1) meaning of visual disability differs between the intent of the question and assigned response; or (2) meaning of visual disability differs between the person's view and assigned response.

The first matters most for policy creation and the latter when trying to understand how individuals evaluate their abilities to navigate the physical world. In this paper, when an allocation is said to differ from the "truth," it refers to the first condition-where the assigned response incorrectly measures disability status relative to the intent of the question. The main point is that allocations have the potential to introduce measurement error in the assessment of disability. Because allocated responses may differ from the truth, investigating rates of allocation by mode is crucial as we seek to advance our understanding in the quality of disability prevalence estimates.

\subsection{Inter-decennial Census}

In 1976, the US Congress enacted an amendment that directed federal agencies to make use of "the most recently produced and published data" to determine how to distribute funds to local governments (Kish, 1981). The legal requirement to obtain "mid-decade" estimates (Torrieri, 2007) on the characteristics of the US population gained momentum after the 1990 US decennial Census had a lower than expected response rate. Leslie Kish, a professor at the Institute for Social Research at the University of Michigan, had previously proposed the use of "cumulated rolling samples"- the approach was argued to be able to provide more timely, accurate, and useful estimates than decennial census - during the 1980s when the need to produce mid-decade estimates was first being explored (Kish, 1981). The approach was primarily challenged by questioning the US Census Bureau's ability to develop a permanent and professional staff capable of meeting the needs of such a sophisticated method. Until now, no publication has shown yearly turn-over rates in ACS personnel, qualifications for interviewers, or the development of matrices for evaluating their performance relative to the production of high quality data. Despite early and current reservations, the ACS, the 
inter-decennial census in the US, was born from the idea that "continuous measurement" was possible by use of a rolling sample (Torrieri, 2007).

Operational feasibility began to be plan in the 1990s and by 1996 the ACS was tested in four counties and over many $(n=1,000)$ in 2000 before entering full implementation in 2005 . Disability information on the US is no longer collected during the decennial census. Information on disability, post-2000, is only available from ACS as pre-tabulated tables or through Public Use Microdata Sample (PUMS) files-which provide individual record (person or household) information. A notable difference between the decennial and ACS is that the first uses "usual residence" and the latter "current residence" to determine place of residence.

The primary goal for ACS data is to provide estimates on the characteristics of the US population. ACS data need only provide an understandable and acceptably valid glimpse on the condition of the population-i.e., a blurry but informative picture of the landscape. The ACS is only meant to provide characteristics of the population and not counts (Alexander, 2002). Hypothetically and by way of example, ACS data only needs to provide some evidence that about $10 \%$ of the population has difficulty with independent-living (e.g., shopping without assistance) and is not required to precisely report that $31,962,847$ individuals experienced this form of disability in the year 2014. Please note previous publications have discussed the need to pay attention to the quality of estimates (Siordia \& Ve, 2013) for "small population groups" (Alexander, 2002). For example, ACS data need only estimate the disability rate in Native Americans and not the actual count of disable in their group who reside in the state of Texas.

The decennial census provides the full count of the US population. In contrast, the ACS was created to describe the characteristics of communities in the US. ACS data helps decisions by governmental agencies to be informed by "changing conditions" rather than the "historical changes" that occurred over the past decade (Alexander, 2002). Because ACS data can affect how individuals in need (e.g., those with disability) receive services, the public health sector should contribute towards giving the ACS more visibility in the hopes that it will foster more civic participation with the survey.

\subsection{Allocations}

Disability data from ACS is used to characterize the disable population in the US and to help federal agencies meet the requirements set by the Americans with Disability Act. In order to achieve the most complete data, the ACS uses "minimal questionnaire completeness" where all "100\% items" (e.g., age, sex, etc.) must be available for the survey not to be discarded as a blank response (i.e., no response) - if a survey has too many empty responses it is not considered further. An "acceptability index" is used by the US Census Bureau to determine if there is enough information within-household to keep the survey.

Some of the surveys passing the necessary acceptability index threshold have missing or "inconsistent" information for some of the survey items (i.e., questions). If there is no response for a survey question, it is said that the item is "missing." If there is a response but it 
is deemed as illogical given some computer algorithm created by survey statisticians, then it is said that the item is "inconsistent." For example, if an immigrant reports to have entered the US at the age of 40 and then reports to only be 30 years in age, the response to the "year of entry' question is deemed illogical since age is a $100 \%$ item.

Both missing and illogical answers can be fixed through "assignments." Please note that assignments use one or more responses to other questions to infer what the missing response could be for the missing or illogical response. Using a set of responses to infer others makes use of a series of assumptions which have not been clearly divulge or defended in a public sphere. Assignments are non-probability based data editing approaches that involve the use of an explicitly logic written as a computer algorithm (Siordia \& Young, 2013).

The logic informing the algorithm is determined by programmers who presumably consult the academic literature and experts in the related field for the specific topic. Until now, no publication has dissected the assumptions in the algorithms used to assign disability responses. Allocations use both non-probability and probability based approaches. Allocations are said to have used probability based methods when they develop a pool of potential "donors" (i.e., other participants matching on a set of factors whose response may be selected for the "recipient" person) through hot-deck or cold-deck matrices (Siordia \& Young, 2013). Others have explained the use of hot deck imputation to impute long form data at the US Census Bureau (Kim \& Fuller 2004).

Why do the methods or rates of allocation matter for estimating disability prevalence? Estimates have confidence intervals around them-e.g., where the true estimate is said to exist somewhere between the upper and lower 99\% confidence limits. Estimating the variance (i.e., width of confidence intervals) around disability prevalence estimates only takes into account the probability of selection into the sample. Equally important is the uncertainty around the estimate potentially created by imputation and the raking to census population controls (discussed below).

From this view, allocations are framed as a non-probability approach - even when they involve the use of the nearest neighbor household (i.e., other participants in geographical proximity whose response may be selected for the recipient) to allocate a response. Other researchers have explained how allocations are made using neighbors (Chen \& Shao, 2000; Kim et al., 2008, 2011; Kim \& Fuller, 2004) and appropriate variance estimation with such procedures (Shao and Wang, 2008; Shao, 2009). The main point is that popular methods for calculating confidence intervals around sample derived estimates may underestimate the width of intervals as they typically ignore potential contamination of the estimate by non-random phenomenon (e.g., item missingness not missing completely at random). Rate of allocation, as measured by "within-person item-completeness" (WPIC), may help understand how the potential for estimate contamination varies as a function of demographic factors. WIPC quantification in disability items is the focus of the current study.

The ACS microdata being used in this study provides an "allocation flag" variable to identify which items were allocated for a particular person (or housing unit). WPIC will be calculated using these allocation flag variables in the microdata. Even after an extensive search, it 
appears from the published data by the US Census Bureau - the federal agency charged with the administration of ACS - that these allocation flags include assigned responses. The only statement of the topic explains that "flag" variables include both assignments and allocations (Schartz, 2007). The same author explains that public use data does not allow researchers the ability to detect when an item was assigned (Schartz, 2007).

For the remainder of this paper, it will be assume that the "allocation flag" variable refers to both assignments and allocations based on either non-probability or probability methods. WPIC is framed as a useful and proxy measure of unaccounted bias in the estimate-a justifiable approach as others have explained that "item allocation rates are final measures of completeness that quantify how frequently allocation was the source of data in the production" of a particular estimate (McGovern \& Griffin, 2003). By focusing on item imputation, this study seeks to investigate if potential measurement error varies as a function of demographic characteristics.

Methods used by the US Census Bureau are widely accepted within the academic field. Methods used in the production of ACS data can be critique only because they continue to be more and more forthright about the procedures employed to produce complete datasets. Few large studies in the academic world provide such a wealth of information and dare to risk face validity in such a way. It is only because the US Census Bureau is so transparent and truly interested in improving their methods that this paper and all its critiques are possible. There is no better data set available for estimating disability prevalence in the US than the ACS.

\subsection{Bias in Estimates}

Survey studies are challenged by the fact that some individuals refuse to participate with the study (referred to as "person-nonresponse") and that from those who do participate, not all answer every question (referred to as "item-nonresponse"). Sampling error, the quantifiable variance associate with creating estimates from a sample instead of the complete population, is different than "non-sampling error"-which is difficult to quantify as it refers to such things as: person nonresponse; item nonresponse; errors of coverage; measurement (i.e., questions are misunderstood); and data processing (Salvo \& Lobo, 2003). Person-nonresponse is said to have the potential to bias estimates when the probability of becoming a study participant is related with the phenomenon of interest. If we assume person nonresponse is probabilistic for the full sample, then we could formulae:

$$
\text { Bias }=\operatorname{Cov}\left(r_{i}, Y_{i}\right)-E\left(p_{n}\right) \bar{Y}
$$

where $\operatorname{Cov}\left(r_{i}, Y_{i}\right)$ is the covariance between the probability of becoming a respondent $\left(r_{i}\right)$ and the values of the variable of interest $\left(Y_{i}\right)$ minus the product of overall mean of variable of interest $(\bar{Y})$ and the expected person nonresponse rate $E\left(p_{n}\right)$ (Lessler \& Kalsbeel, 1992). We may be able to extend this formula to conceptually represent how item nonresponse and mode may affect bias:

$$
\text { Bias }=\left[\operatorname{Cov}\left(r_{i}, Y_{i}\right)-E\left(p_{n}\right) \bar{Y}\right]+\left[\operatorname{Cov}\left(s_{i}, R_{i}\right)-E\left(i_{n}\right) \bar{R}\right]+\left[\operatorname{Cov}\left(m_{i}, D_{i}\right)-E\left(o_{n}\right) \bar{D}\right]
$$


where $\operatorname{Cov}\left(s_{i}, Y_{i}\right)$ is the covariance between the probability of answering the survey item within responders $\left(s_{i}\right)$ and the values of the variable $\left(R_{i}\right)$ minus the product of overall mean $(\bar{R})$ and the expected item nonresponse rate within responders $E\left(i_{n}\right)$, and where $\operatorname{Cov}\left(m_{i}, D_{i}\right)$ is the covariance between the probability of answering the survey item via MAIL mode $\left(m_{i}\right)$ and the values of the variable $\left(D_{i}\right)$ minus the product of overall mean $(\bar{D})$ and the expected item nonresponse rate within MAIL participants $E\left(o_{n}\right)$. In short, item nonresponse and mode of data collection may have the potential to add bias in the estimate.

Among survey researchers it is an article of faith that high person- and item-response rates are better than low response rates (Tourangeau, 2004). What constitutes "high" is debatable. The need for high response rates is based on the assumption that high person- and/or item-nonresponse have the ability to make the estimation of reliability in estimates impossible. As a consequence to these issues, the search for producing complete data (i.e., high response rate data banks with no missing information) has driven sophisticated studies like the ACS to use "sequential multi-mode systems" (de Leeuw, 2005) to help reduce both person- and item-nonresponse error. Others have recently reviewed issues with unit nonresponse and weighting adjustments in models (Brick, 2013). The main point here is that confidence intervals around disability prevalence may not fully capture the full level of uncertainty around the estimate.

\subsection{Specific Aim}

The specific aim of this technical report is to present the allocation rates for disability items in the ACS by mode of data collection. Presumably, the rates will identify if potential measurement bias and contamination of estimate varies by mode along various demographic characteristics. The report fills the gap in public health survey methodology literature on estimating disability prevalence by introducing the reader to easy-to-follow estimates of allocations in disability items and discussing how these patterns may affect estimates of disability prevalence in the US.

\section{Methods}

\subsection{Data}

The source of the data under analysis has been discussed at length by now. Here, it is simply mentioned more technically that rates of allocation were produced using ACS PUMS 3-year files from 2009 to 2011 . The ACS is a population based yearly study administered by the US Census Bureau and is intended to be used by the US federal government to help distribute billions of dollars in federal and state funds (Reamer, 2010). The 3-year file collects data over an approximately 36 month period.

The ACS is the most reliable data source for developing "period estimates" of disability prevalence in the US. They must be referred to as period estimates because the sample is made up of different years - where presumably, all individuals re-enter the sampling frame on a yearly basis. Detailed documentation on this from the US Census Bureau is needed. The 3-year public use file provides a sample of more than 9 million people said to represent the more than 300 million people in the US. By contrast, some of the most widely used studies claiming to 
ascertain disability prevalence in the US population only make use of a few hundred people or a few thousand subjects - selected from a smaller geographical area and through less sophisticated sampling protocols than those found in the ACS. The public health research sector should seek ways to make more wide use of this incomparable data source.

\subsection{Sample}

Actual counts, real number of people in sample, are referred to as "unweighted" counts. In contrast, representative counts (i.e., inferred number of people in sample), are referred to as "weighted" counts. Weighted counts are estimate by applying the population weight (PWGTP variable) to the person- unit. A population weight is a variable said to indicate how many people are represented by one person and may be represented with the following formula:

$$
\text { Weithed Count }=\left(S_{i} \times P_{w_{i}}\right)
$$

where the weighed count is the product of $\mathrm{i}^{\text {th }}$ subject $\left(S_{i}\right)$ and his/her population weight $\left(P_{w_{i}}\right)$. For example, assume person-A has a population weight of 26 , then: $(1 \times 26=26)$-meaning that person-A represents about 26 of his/her peers in the general population. The characteristics of person-A will be used to infer the characteristics of another 26 people in the population.

Describing how population weights are produced by ACS administrators is beyond the scope of the current study. Others have explained the topic using US Census 2000 decennial data (Kim et al., 2011). Briefly, they are produced through sample-balancing which may be achieved by using iterative post-stratification by geography to match distributions of a survey sample with decennial counts through a process known as "raking." Raking can be done by multiple variables and categories. Since ACS is a sample, the individuals in the data only cover a segment of the "target population" in proportions that do not match those of the population. In the ACS, the decennial counts represent the "source of control totals." ACS counts are assigned population weights by raking them up to the decennial counts. More formally and conceptually, population weighting could be formulated as follows:

$$
w_{h}=\left(\frac{r P_{h}}{r_{h}}\right)
$$

where $w_{h}$ is a quotient representing the post-stratification weight " $w$ " for each sample case in post-stratum " $h$ "; $r P_{h}$ is the product of " $r$ " is the respondent sample size and $P_{h}$-the population source control; divided by $r_{h}$ is the number of survey respondents " $r$ " in post-stratum " $h$ ". Survey estimates, like disability prevalence, would weight cases by $w_{h}$. More appropriate estimations are possible (Little, 1993).

The use of raking in federal data merits more attention (Battaglia et al., 2004). Please note that after a careful investigation, no public data source was found to clarify if the ACS is raked up to 'actual' decennial counts or if they are raked up to inter-decennial statistically derived counts that have been projected out from decennial counts. For example, sample design or person nonresponse may create a sample for a particular geography (e.g., county) 
with only $20 \%$ males although count data from decennial census shows the county is $40 \%$ male.

Under this hypothetical and oversimplified scenario, the males in the sample would have larger population weights than females to make the county match the decennial numbers. This is done over many variables and by different geographies-with multi-way-tables. If "hot" raking is unable to balance the sample (i.e., converge of raking algorithm is not obtained through statistical iterations), "cold" not-geographically referenced and multi-way-tables with less dimensions are then used. The US Census Bureau should also document if "weight truncation" (lowering sampling variability by lowering high weight values) or "weight trimming" (reducing means squared error of the key outcome estimates) is used in their raking procedures (Srinath, 2003).

Although the topic of raking has been around for many decades (Deming, 1943; Bishop et al., 1975) and received attention in the 1990s (Deville \& Särndal, 1992; Deville et al., 1993) and 2000s (Battaglia et al., 2004; Brick et al., 2003; Lumley et al., 2004), not enough consideration has been given to the implications of these procedures in producing population weights in ACS. In order to highlight the average number of people being represented in weighted population by the unweighted counts, the Person Inflation Ratio (PIR) is calculated using the following formula:

$$
P I R=\left[\left(\frac{W_{y_{g}}}{u W_{t y_{g}}}\right)\right]
$$

where PRI is the quotient between $W_{y_{g}}$, the weighted "W" count of variable " $\mathrm{y}$ " in "g" group, and $u W_{t y_{g}}$, the unweighted ("uW") total "t" of variable "y" in group "g."

For example, in Table 6 below we see the weighted count of Non-Latino-Blacks participating via MAIL is $12,366,536$ and the unweighted count is $441,636:(12,366,536 \div 441,636)=28$. This means that Non-Latino-Blacks participating via MAIL mode on average represent about 28 of their Non-Latino-Black counterpart participating via MAIL mode. Please not PIR is an average over the group and that representation could range from 1 all the way to hundreds or thousands of people. Ideas are offered in closing for future research on this topic. Please note this level of detail is only possible tanks to the US Census Bureau's interest in engaging the research community as they continue to make every effort in providing the US Congress with the most up-to-date and reliable disability estimates of the US population.

\subsection{Disability}

Concepts of disability where first included in a decennial census in 1830 (asked if person was "blind," "deaf," or "mute") and the term "disability" was first used in the 1880 decennial census, dropped out of census forms in the early 1900s, and returned into content in 1970 (Brault et al., 2007). Concerns raised with the 1970 questions led the Committee on Disability and Health of the Federal Agency Council (DHF AC) to request that the "disability spectrum" 
be broadened. National Content Test (NCT) for disability items were tested in 1976 and again in 1986 after the 1980 decennial census included a question about limitations with using public transportation. By 1990, questions about work disability (in 1970) and transportation limitation (in 1980) were expanded to include questions about limitations with going outside alone and taking care of personal needs-leading to a 1996 National Content Survey (NCS).

By 1997, the Subcommittee on Disability Statistics and the US Census Bureau found the questions to be lacking and advised the Office of Management and Budget (OMB) that more detailed questions were needed-where the sensory, physical, and cognitive domains would need to be explored. These questions were introduced in the 2000 decennial long-form Census. Because the US Census Bureau will only collect legally required data on the US population, the readers should note the primary purpose of disability information in the ACS if to help with "provision of services" and opportunities in housing, education, and other areas. Disability is loosely defined by survey administrators as the restriction in participation that results from a lack of fit between the individual's functional limitations and the characteristics of their environment.

Questions make use of the Institute of Medicine (IOM) Model of Disability and the International Classification of Functioning (ICF) Model of Disability to derive their definitions. The National Center for Health Statistics (NCHS) and the US Census Bureau have undertaken cognitive testing of disability items. Others have explained that in survey research, disability should be seen as extrinsic to the individual and attempts should be made to capture disability by asking about the components that make up the process (Brault et al., 2007). Disability questions in the ACS are not meant as a diagnostic tool or as a way of understanding the severity or cause of a disabling condition.

For a full description of disability items in the ACS please see Siordia and Young (2013). Briefly: "self-care" refers to reported difficulty with outdoor activities like shopping; "independent" refers to reported difficulty with basic indoor activities like bathing; "ambulatory" refers to reported difficulty with indoor activities like climbing stairs; "cognitive" refers to reported difficulty with cognition; "hearing" refers to reported auditory difficulty; and "vision" refers to reported visual difficulty. These are all the disability items found in the ACS. Allocation rates for each of them are provided by demographic characteristics and mode of data collection.

\subsection{Quantifying Allocations}

Both unweighted and weighted allocation rates are presented as a count and percent in the tables below. The tables show the "universe" for the demographic variables. The universe refers to the total number of weighed individuals represented in the table. All the tables divide individuals into one of two groups (e.g., female or male) and by mode (i.e., MAIL vs. CAPI/CATI). The unweighted number of allocations by demographic characteristic and mode is presented after showing the reader the "unweighted count" as "UN=" (unweighted sample) and the "weighed count" as "WN=" (weighed sample) - these numbers represent the denominators used to calculate the percent of allocation. More technically, the "unweighted percent allocated" (UPA) is calculated as follows: 


$$
U P A=\left[\left(\frac{u W_{x_{a}}}{u W_{t x_{a}}}\right) \times 100\right]
$$

where unweighted percent allocated is the quotient between $u W_{x_{a}}$, the unweighted allocated count of " $\mathrm{x}$ " item for group "a," and $u W_{t x_{a}}$, the unweighted total of $\mathrm{x}$ item of those in group "a." For example, from Table 1, we see that in MAIL mode, females Self-Care has 108,388 unweighted allocations and that there are 3,116,347 unweighted females: (108,388 $\div$ $3,116,347=0.03478 \times 100=3.48 \%$ ). If we extend the previous equation, the "weighted percent allocated" (WPA) is calculated as follows:

$$
W P A=\left[\left(\frac{W_{x_{a}}}{W_{t x_{a}}}\right) \times 100\right]
$$

where weighed percent allocated is the quotient between $W_{x_{a}}$, the weighted allocated count of "x" item for group "a," and $W_{t x_{a}}$, the weighted total of $\mathrm{x}$ item of those in group "a." For example, from Table 2, we see that in CAPI/CATI mode, individuals with " $\leq$ no college" have 2,000,690 weighted allocations for the Ambulatory disability item and that there are $83,758,331$ weighted individuals whit no college or less: $(2,000,690 \div 83,758,331=0.0239 \times$ $100=2.39 \%$ ). The reason why both unweighted and weighed allocation rates are presented is to show readers instances in CATI/CAPI when population weights "shrink" or "deflate" the allocation rate. The fact that population weights have the ability to deflate estimated allocation rates in CATI/CAPI but not in MAIL mode for minority groups (e.g., Mexican-Latinos) may merit special attention as the numbers may indicate that population weights in minorities allocated in CATI/CAPI mode have smaller population weights than those used with minorities not allocate during CATI/CAPI. An explanation of whether weights vary systematically is beyond the scope of the current study and suggestions for future work are suggested in closing.

\subsection{Demographics}

Individuals are separated over basic demographic variables: females vs. males; those with no college education or below vs. those with some college education and beyond; those in households who only speak Spanish vs. those in households where English is the only language; and those in married-couple households vs. those in non-married-couple households (e.g., singles, single-father, etc.). Race-ethnicity groupings are also used as follows: Mexican-Latino; Non-Latino-White; Non-Latino-Black; and Non-Mexican-Latinos (e.g., Salvadorians, Hondurans, Puerto Ricans, etc.). Note Non-Latino-Other (e.g., Native Americans, Asians, etc.) is an omitted contrast group. Details on race and ethnicity are made widely available by the US Census Bureau online. The employed coding scheme has been used before because it makes use of population size to represent the largest race-ethnic groups in the US. 


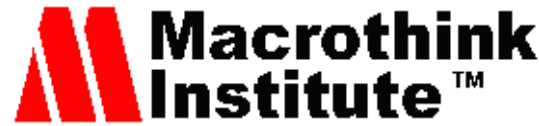

\section{Results}

\subsection{Females vs. Males}

The reader can digest the tables in many different ways, as multiple and complex comparisons are possible with the detailed tabulation provided in the report. In order to simply highlight some of the most notable facts all the results are interpreted following a similar pattern. If we compare the allocation rates in the weighted counts between MAIL to CAPIT/CATI in Table 1 and within females, we see allocations were more prevalent in MAIL - the same is true for males. When compared to males, females have slight lower allocations rates over all the six disability related items. Within the disability items, Vision seems to have the most allocation rates for both males and females.

Table 1. Unweighted and weighted allocations by sex, mode, and disability item (universe $=301,225,855$ )

\begin{tabular}{|c|c|c|c|c|c|c|c|c|}
\hline \multirow[b]{4}{*}{ Self-Care } & \multicolumn{8}{|c|}{ Females } \\
\hline & \multicolumn{4}{|c|}{ Mail $\left(\mathrm{PIR}^{1}=\mathbf{2 6 . 5}\right)$} & \multicolumn{4}{|c|}{ CAPI/CATI $(\mathrm{PIR}=48.3)$} \\
\hline & \multicolumn{2}{|c|}{$\mathrm{U}^{2} \mathrm{~N}=3,116,347$} & \multicolumn{2}{|c|}{$\mathrm{W}^{3} \mathrm{~N}=82,469,416$} & \multicolumn{2}{|c|}{$\mathrm{U}^{4} \mathrm{~N}=1,481,485$} & \multicolumn{2}{|c|}{$\mathrm{W}^{5} \mathrm{~N}=71,593,569$} \\
\hline & 108,388 & $3.48 \%$ & $2,874,556$ & $3.49 \%$ & 30,549 & $2.06 \%$ & $1,529,526$ & $2.14 \%$ \\
\hline Independent & 92,820 & $2.98 \%$ & $2,472,882$ & $3.00 \%$ & 25,265 & $1.71 \%$ & $1,291,195$ & $1.80 \%$ \\
\hline Ambulatory & 108,968 & $3.50 \%$ & $2,888,821$ & $3.50 \%$ & 30,418 & $2.05 \%$ & $1,523,764$ & $2.13 \%$ \\
\hline Cognitive & 107,110 & $3.44 \%$ & $2,838,158$ & $3.44 \%$ & 31,025 & $2.09 \%$ & $1,549,304$ & $2.16 \%$ \\
\hline Hearing & 98,244 & $3.15 \%$ & $2,610,654$ & $3.17 \%$ & 31,695 & $2.14 \%$ & $1,581,434$ & $2.21 \%$ \\
\hline \multirow[t]{4}{*}{ Vision } & 112,812 & $3.62 \%$ & $2,985,708$ & $3.62 \%$ & 31,951 & $2.16 \%$ & $1,592,007$ & $2.22 \%$ \\
\hline & \multicolumn{8}{|c|}{ Males } \\
\hline & \multicolumn{4}{|c|}{ Mail $(P I R=26.8)$} & \multicolumn{4}{|c|}{ CATI/CAPI $(P I R=49.8)$} \\
\hline & \multicolumn{2}{|c|}{$\mathrm{U}^{6} \mathrm{~N}=2,873,383$} & \multicolumn{2}{|c|}{$\mathrm{W}^{7} \mathrm{~N}=77,030,844$} & \multicolumn{2}{|c|}{$\mathrm{U}^{8} \mathrm{~N}=1,408,385$} & \multicolumn{2}{|c|}{$\mathrm{W}^{9} \mathrm{~N}=70,132,026$} \\
\hline Self-Care & 100,637 & $3.50 \%$ & $2,730,619$ & $3.54 \%$ & 31,810 & $2.26 \%$ & $1,591,733$ & $2.27 \%$ \\
\hline Independent & 83,211 & $2.90 \%$ & $2,278,247$ & $2.96 \%$ & 26,339 & $1.87 \%$ & $1,345,454$ & $1.92 \%$ \\
\hline Ambulatory & 100,784 & $3.51 \%$ & $2,733,333$ & $3.55 \%$ & 31,799 & $2.26 \%$ & $1,590,740$ & $2.27 \%$ \\
\hline Cognitive & 99,543 & $3.46 \%$ & $2,699,845$ & $3.50 \%$ & 32,637 & $2.32 \%$ & $1,626,904$ & $2.32 \%$ \\
\hline Hearing & 96,646 & $3.29 \%$ & $2,562,936$ & $3.33 \%$ & 33,284 & $2.36 \%$ & $1,655,699$ & $2.36 \%$ \\
\hline Vision & 110,590 & $3.85 \%$ & $2,970,459$ & $3.86 \%$ & 33,471 & $2.38 \%$ & $1,666,425$ & $2.38 \%$ \\
\hline
\end{tabular}

${ }^{1}$ Person Inflation Ratio $=$ (weighted count $\div$ weighted total population $) ;{ }^{2}$ Unweighted number of females participating via MAIL; ${ }^{3}$ Weighted number of females participating via MAIL; ${ }^{4}$ Unweighted number of females participating via CATI/CAPI; ${ }^{5}$ Weighted number of females participating via CATI/CAPI; ${ }^{6}$ Unweighted number of males participating via MAIL; ${ }^{7}$ Weighted number of males participating via MAIL; ${ }^{8}$ Unweighted number of males participating via CATI/CAPI; ${ }^{9}$ Weighted number of males participating via CATI/CAPI

The most notable difference on the rate of allocation caused by using population weights is found in the Independent item for females where the unweighted allocation rate is $1.71 \%$ and the weighted allocation rate is $1.80 \%$ - only a $0.09 \%$ increase. In general, weighted allocation rates by sex range from a low $1.80 \%$ to $3.86 \%$ - suggesting a high ( $\geq 96 \%)$ level of completeness for disability items by sex and mode. A crucial finding from Table 1 is that MAIL has a PIR of about 26 compare to the CAPI/CATI PIR of abo ut 49 -indicating that on 


\section{Macrothink Mnstitutem}

average, individuals participating with the survey via mails represent fewer of their counterparts than those who participate via telephone or in-person interviews. This matters if mode of interview has the ability to influence response editing (e.g., changing answer to question because of social desirability).

\subsection{No College vs. Some College}

Table 2 shows that allocation rates from weighted counts between MAIL to CAPIT/CATI and within those with less than a college education, we see allocations were most prevalent in MAIL - a difference much less pronounce within the group with some college education and beyond. This may indicate the rate of item completeness by mode varies most when low or moderate levels of education are present. When compared to tho se with some college or more, those with no college had higher rates of allocation in MAIL but only a slight increase in CAPI/CATI. Within the disability items, Vision in general has the most allocations.

Table 2. Unweighted and weighted allocations by educational attainment, mode, and disability item (universe $=289,376,193$ )

\begin{tabular}{|c|c|c|c|c|c|c|c|c|}
\hline \multirow[b]{4}{*}{ Self-Care } & \multicolumn{8}{|c|}{$\leq$ No College } \\
\hline & \multicolumn{4}{|c|}{ Mail $\left(P^{\prime}{ }^{1}=26.1\right)$} & \multicolumn{4}{|c|}{ CAPI/CATI $(P I R=48.0)$} \\
\hline & \multicolumn{2}{|c|}{$\mathrm{U}^{2} \mathrm{~N}=2,892,563$} & \multicolumn{2}{|c|}{$\mathrm{W}^{3} \mathrm{~N}=75,563,715$} & \multicolumn{2}{|c|}{$\mathrm{U}^{4} \mathrm{~N}=1,743,712$} & \multicolumn{2}{|c|}{$\mathrm{W}^{5} \mathrm{~N}=83,758,331$} \\
\hline & 137,661 & $4.76 \%$ & $3,633,045$ & $4.81 \%$ & 41,766 & $2.40 \%$ & $2,004,890$ & $2.39 \%$ \\
\hline Independent & 103,179 & $3.57 \%$ & $2,737,502$ & $3.62 \%$ & 30,715 & $1.76 \%$ & $1,506,366$ & $1.80 \%$ \\
\hline Ambulatory & 138,053 & $4.77 \%$ & $3,641,279$ & $4.82 \%$ & 41,683 & $2.39 \%$ & $2,000,690$ & $2.39 \%$ \\
\hline Cognitive & 136,585 & $4.72 \%$ & $3,602,252$ & $4.77 \%$ & 42,624 & $2.44 \%$ & $2,038,907$ & $2.43 \%$ \\
\hline Hearing & 123,600 & $4.27 \%$ & $3,265,420$ & $4.32 \%$ & 42,386 & $2.43 \%$ & $2,028,853$ & $2.42 \%$ \\
\hline \multirow[t]{4}{*}{ Vision } & 138,382 & $4.78 \%$ & $3,637,337$ & $4.81 \%$ & 42,611 & $2.44 \%$ & $2,038,495$ & $2.43 \%$ \\
\hline & \multicolumn{8}{|c|}{$\geq$ Some College } \\
\hline & \multicolumn{4}{|c|}{ Mail (PIR=27.1) } & \multicolumn{4}{|c|}{ CATI/CAPI $(P I R=50.2)$} \\
\hline & \multicolumn{2}{|c|}{$\mathrm{U}^{6} \mathrm{~N}=2,910,379$} & \multicolumn{2}{|c|}{$\mathrm{W}^{7} \mathrm{~N}=78,798,024$} & \multicolumn{2}{|c|}{$\mathrm{U}^{8} \mathrm{~N}=1,021,490$} & \multicolumn{2}{|c|}{$\mathrm{W}^{9} \mathrm{~N}=51,256,123$} \\
\hline Self-Care & 71,364 & $2.45 \%$ & $1,972,130$ & $2.50 \%$ & 20,593 & $2.02 \%$ & $1,116,369$ & $2.18 \%$ \\
\hline Independent & 72,852 & $2.50 \%$ & $2,013,627$ & $2.56 \%$ & 20,889 & $2.05 \%$ & $1,130,283$ & $2.21 \%$ \\
\hline Ambulatory & 71,699 & $2.46 \%$ & $1,980,875$ & $2.51 \%$ & 20,534 & $2.01 \%$ & $1,113,814$ & $2.17 \%$ \\
\hline Cognitive & 70,068 & $2.41 \%$ & $1,935,751$ & $2.46 \%$ & 21,038 & $2.06 \%$ & $1,137,301$ & $2.22 \%$ \\
\hline Hearing & 54,726 & $1.88 \%$ & $1,518,088$ & $1.93 \%$ & 19,531 & $1.91 \%$ & $1,063,012$ & $2.07 \%$ \\
\hline Vision & 70,167 & $2.41 \%$ & $1,921,032$ & $2.44 \%$ & 19,756 & $1.93 \%$ & $1,075,292$ & $2.10 \%$ \\
\hline
\end{tabular}

\footnotetext{
${ }^{1}$ Person Inflation Ratio $=($ weighted count $\div$ weighted total population $) ;{ }^{2}$ Unweighted number of $\leq$ no college participating via MAIL; ${ }^{3}$ Weighted number of $\leq$ no college participating via MAIL; ${ }^{4}$ Unweighted number of $\leq$ no college participating via CATI/CAPI; ${ }^{5}$ Weighted number of $\leq$ no college participating via CATI/CAPI; ${ }^{6}$ Unweighted number with $\geq$ some college participating via MAIL; ${ }^{7}$ Weighted number with $\geq$ some college participating via MAIL; ${ }^{8}$ Unweighted number with $\geq$ some college participating via CATI/CAPI; ${ }^{9}$ Weighted number with $\geq$ some college males participating via CATI/CAPI
} 


\subsection{Spanish vs. English only Households}

Table 3 shows that allocation rates almost triple from CATI/CAPI to MAIL with weighted counts and within households where language used is "Spanish only" - a difference that remains for "English only" households although much less pronounced. When compared to English only language household, Spanish only household in general have much higher rates of allocation in mail but lower rates in CATI/CAPI. As before, Vision has the most allocation rates and Vision, in English only households for MAIL, has a deflation of $1.43 \%$ on the allocation rate when population weights are applied. In general, weighted allocation rates by language used at home ranges from a low $1.53 \%$ to $6.39 \%$ - suggesting a high ( $\geq 93 \%$ ) level of completeness for disability items by language and mode. As with Table 1 and 2, PIR scores are almost double from MAIL to CAPI/CATI.

Table 3. Unweighted and weighted allocations by household language, mode, and disability item (universe $=270,432,061$ )

\begin{tabular}{|c|c|c|c|c|c|c|c|c|}
\hline \multirow[b]{4}{*}{ Self-Care } & \multicolumn{8}{|c|}{ Household Language Spanish Only } \\
\hline & \multicolumn{4}{|c|}{ Mail (PIR $\left.{ }^{1}=27.0\right)$} & \multicolumn{4}{|c|}{ CAPI/CATI $(P I R=51.8)$} \\
\hline & \multicolumn{2}{|c|}{$U^{2} N=566,242$} & \multicolumn{2}{|c|}{$W^{3} N=15,296,343$} & \multicolumn{2}{|c|}{$\mathrm{U}^{4} \mathrm{~N}=633,649$} & \multicolumn{2}{|c|}{$\mathrm{W}^{5} \mathrm{~N}=32,841,707$} \\
\hline & 34,382 & $6.07 \%$ & 945,862 & $6.18 \%$ & 13,522 & $2.13 \%$ & 615,673 & $1.87 \%$ \\
\hline Independent & 26,380 & $4.66 \%$ & 739,818 & $4.84 \%$ & 10,621 & $1.68 \%$ & 503,688 & $1.53 \%$ \\
\hline Ambulatory & 34,418 & $6.08 \%$ & 945,866 & $6.18 \%$ & 13,497 & $2.13 \%$ & 614,078 & $1.87 \%$ \\
\hline Cognitive & 33,981 & $6.00 \%$ & 933,335 & $6.10 \%$ & 13,697 & $2.16 \%$ & 622,092 & $1.89 \%$ \\
\hline Hearing & 33741 & $5.96 \%$ & 925,443 & $6.05 \%$ & 14741 & $2.33 \%$ & 662,328 & $2.02 \%$ \\
\hline \multirow[t]{4}{*}{ Vision } & 35,717 & $6.31 \%$ & 978,138 & $6.39 \%$ & 14,769 & $2.33 \%$ & 664,107 & $2.02 \%$ \\
\hline & \multicolumn{8}{|c|}{ Household Language English Only } \\
\hline & \multicolumn{4}{|c|}{ Mail (PIR=26.4) } & \multicolumn{4}{|c|}{ CATI/CAPI (PIR=47.9) } \\
\hline & \multicolumn{2}{|c|}{$\mathrm{U}^{6} \mathrm{~N}=4,797,696$} & \multicolumn{2}{|c|}{$W^{7} \mathrm{~N}=126,608,864$} & \multicolumn{2}{|c|}{$\mathrm{U}^{8} \mathrm{~N}=1,996,309$} & \multicolumn{2}{|c|}{$W^{9} N=95,686,147$} \\
\hline Self-Care & 143,707 & $3.00 \%$ & $3,791,863$ & $2.99 \%$ & 41,626 & $2.09 \%$ & $2,154,316$ & $2.25 \%$ \\
\hline Independent & 125,110 & $2.61 \%$ & $3,310,472$ & $2.61 \%$ & 35,103 & $1.76 \%$ & $1,839,740$ & $1.92 \%$ \\
\hline Ambulatory & 144,242 & $3.01 \%$ & $3,804,939$ & $3.01 \%$ & 41,536 & $2.08 \%$ & $2,150,567$ & $2.25 \%$ \\
\hline Cognitive & 142,006 & $2.96 \%$ & $3,743,571$ & $2.96 \%$ & 42,633 & $2.14 \%$ & $2,198,257$ & $2.30 \%$ \\
\hline Hearing & 131595 & $1.32 \%$ & $3,479,414$ & $2.75 \%$ & 42642 & $2.14 \%$ & $2,205,842$ & $2.31 \%$ \\
\hline Vision & 158,101 & $3.30 \%$ & $4,153,554$ & $3.28 \%$ & 43,017 & $2.15 \%$ & $2,223,709$ & $2.32 \%$ \\
\hline
\end{tabular}

\footnotetext{
${ }^{1}$ Person Inflation Ratio $=($ weighted count $\div$ weighted total population $) ;{ }^{2}$ Unweighted number of participants in Spanish-only HH via MAIL; ${ }^{3}$ Weighted number of participants in Spanish-only HH via MAIL; ${ }^{4}$ Unweighted number of participants in Spanish-only HH via CATI/CAPI; ${ }^{5}$ Weighted number of participants in Spanish-only $\mathrm{HH}$ via CATI/CAPI; ${ }^{6}$ Un weighted nu mber of participants in Eng lish-only HH via MAIL; ${ }^{7}$ Weighted number of participants English-only HH via MAIL; ${ }^{8}$ Unweighted number of participants English-only HH via CATI/CAPI;

${ }^{9}$ Weighted number of participants English-only HH via CATI/CAPI
} 


\subsection{Married-couple vs. Non-married-couple Households}

Table 4 indicates allocation rates are highest amongst non-married-couple family household in MAIL mode. In contrast, married-couple family households have higher rates of allocation in CAPI/CATI than non-married-couple family households. Vision remains the most allocation disability items. A small inflation of $0.10 \%$ on the allocation rate when population weights are applied is present in the Independent variable for married-couple family households. Weighted allocation rates by married-couple type ranges from a low $1.81 \%$ to $4.90 \%$ - suggesting a high $(\geq 95 \%)$ level of completeness for disability items by married-couple family type and mode. As with all the previous tables, PIR scores almost double from MAIL to CAPI/CATI. If bias is more frequently introduced in phone or in-person interviews than in mail responses, any contamination to the reliability of the confidence intervals in the point estimate will propagate due to population weights.

Table 4. Unweighted and weighted allocations by household family type, mode, and disability item (universe $=301,225,855$ )

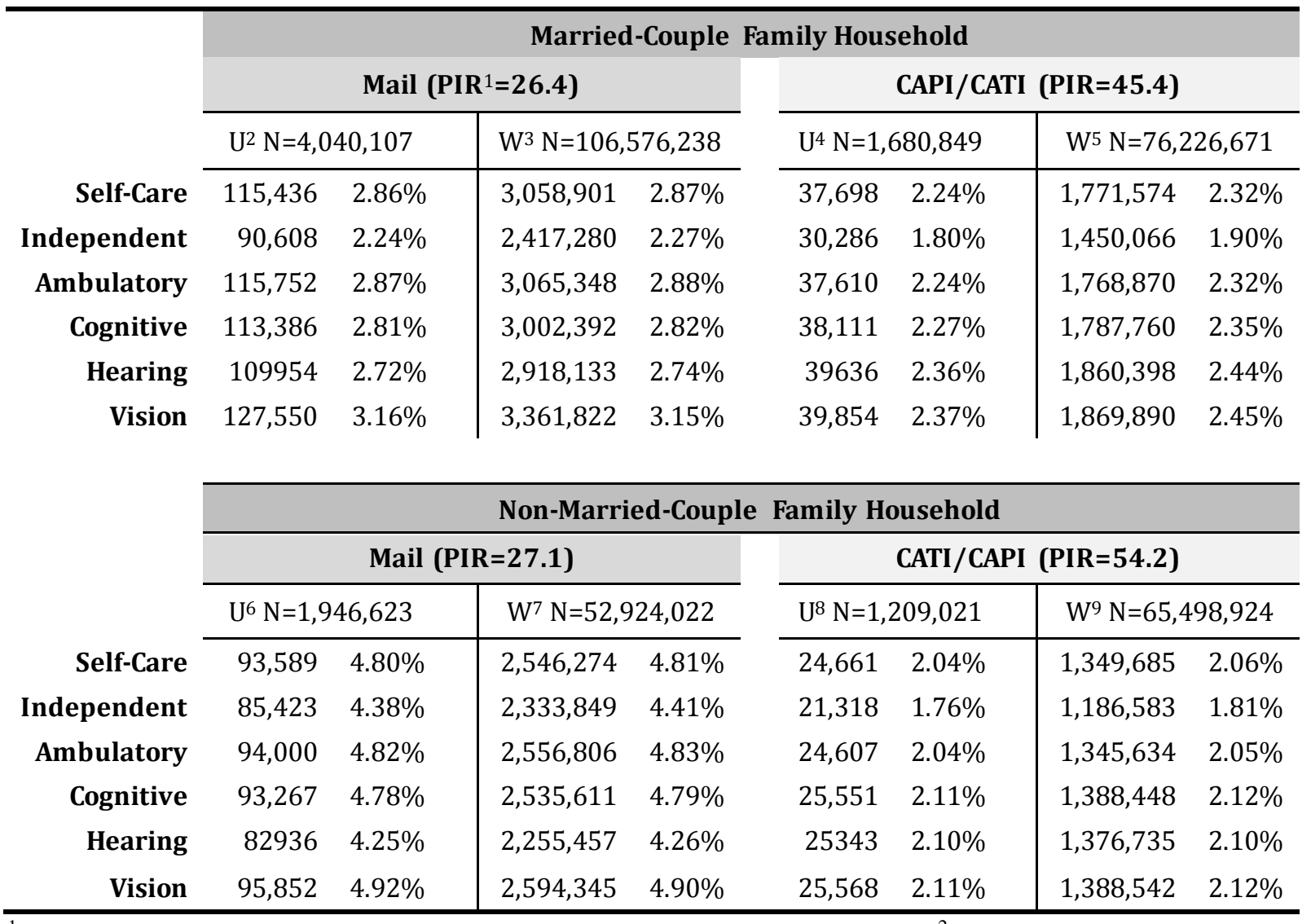

${ }^{1}$ Person Inflation Ratio $=$ (weighted count $\div$ weighted total population); ${ }^{2}$ Unweighted participants in Married-Couple HHs via MAIL; ${ }^{3}$ Weighted participants in Married-Couple HHs via MAIL; ${ }^{4}$ Unweighted participants in Married-Couple HHs via CATI/CAPI; ${ }^{5}$ Weighted participants in Married-Couple HHs via CATI/CAPI; ${ }^{6}$ Unweighted participants in Non-Married-Couple HHs via MAIL; ${ }^{7}$ Weighted participants Non-Married-Couple HHs via MAIL; ${ }^{8}$ Unweighted participants Non-Married-Couple HHs via CATI/CAPI; 9 Weighted participants Non-Married-Couple HHs via CATI/CAPI 


\subsection{Mexican-Latinos vs. Non-Latino-Whites}

Table 5 indicates allocation rates are highest amongst Mexican-Latinos/as than for Non-Latino-Whites only in MAIL mode. In contrast, allocation rates are lower for CAPI/CATI responses in Mexican-Latinos/as than Non-Latino-Whites. Vision remains the most allocation disability items. A small inflation of $0.36 \%$ on the allocation rate when population weights are applied is present in the Hearing and Vision for Mexican-Latinos/as. Weighted allocation rates by race-ethnicity range from a low $1.27 \%$ to $6.24 \%$ - a high ( $\geq$ 93\%) level of completeness for disability items by race-ethnicity and mode. As with all the previous tables, PIR scores almost double from MAIL to CAPI/CATI. Although not shown here, these notable different in inflation ratios by mode may have even larger amplifications if they were observed by geography (e.g., rate of allocation by race, mode, and state).

Table 5. Unweighted and weighted allocations by race-ethnicity, mode, and disability item (universe $=224,471,491$ )

\begin{tabular}{|c|c|c|c|c|c|c|c|c|}
\hline \multirow[b]{4}{*}{ Self-Care } & \multicolumn{8}{|c|}{ Mexican-Latinos/as } \\
\hline & \multicolumn{4}{|c|}{ Mail (PIR = 26.6) } & \multicolumn{4}{|c|}{ CAPI/CATI $(P I R=51.2)$} \\
\hline & \multicolumn{2}{|c|}{$\mathrm{U}^{2} \mathrm{~N}=327,318$} & \multicolumn{2}{|c|}{$\mathrm{W}^{3} \mathrm{~N}=8,703,085$} & \multicolumn{2}{|c|}{$\mathrm{U}^{4} \mathrm{~N}=460,544$} & \multicolumn{2}{|c|}{$\mathrm{W}^{5} \mathrm{~N}=23,600,827$} \\
\hline & 18,976 & $5.80 \%$ & 510,430 & $5.86 \%$ & 8,867 & $1.93 \%$ & 381,683 & $1.62 \%$ \\
\hline Independent & 14,094 & $4.31 \%$ & 386,558 & $4.44 \%$ & 6,702 & $1.46 \%$ & 300,433 & $1.27 \%$ \\
\hline Ambulatory & 19,016 & $5.81 \%$ & 510,651 & $5.87 \%$ & 8,858 & $1.92 \%$ & 381,031 & $1.61 \%$ \\
\hline Cognitive & 18,763 & $5.73 \%$ & 503,650 & $5.79 \%$ & 8,955 & $1.94 \%$ & 384,818 & $1.63 \%$ \\
\hline Hearing & 19079 & $5.83 \%$ & 513,903 & $5.90 \%$ & 9860 & $2.14 \%$ & 420,749 & $1.78 \%$ \\
\hline \multirow[t]{4}{*}{ Vision } & 20,179 & $6.17 \%$ & 543,003 & $6.24 \%$ & 9,882 & $2.15 \%$ & 422,011 & $1.79 \%$ \\
\hline & \multicolumn{8}{|c|}{ Non-Latino-Whites } \\
\hline & \multicolumn{4}{|c|}{ Mail (PIR=26.2) } & \multicolumn{4}{|c|}{ CATI/CAPI (PIR=46.1) } \\
\hline & \multicolumn{2}{|c|}{$\mathrm{U}^{6} \mathrm{~N}=4,564,734$} & \multicolumn{2}{|c|}{$\mathrm{W}^{7} \mathrm{~N}=119,792,201$} & \multicolumn{2}{|c|}{$\mathrm{U}^{8} \mathrm{~N}=1,569,001$} & \multicolumn{2}{|c|}{$W^{9} N=72,375,378$} \\
\hline Self-Care & 124,377 & $2.72 \%$ & $3,245,628$ & $2.71 \%$ & 33,631 & $2.14 \%$ & $1,753,376$ & $2.42 \%$ \\
\hline Independent & 106,859 & $2.34 \%$ & $2,805,263$ & $2.34 \%$ & 28,824 & $1.84 \%$ & $1,523,496$ & $2.11 \%$ \\
\hline Ambulatory & 124,743 & $2.73 \%$ & $3,255,107$ & $2.72 \%$ & 33,539 & $2.14 \%$ & $1,750,169$ & $2.42 \%$ \\
\hline Cognitive & 122,806 & $2.69 \%$ & $3,203,596$ & $2.67 \%$ & 34,368 & $2.19 \%$ & $1,785,071$ & $2.47 \%$ \\
\hline Hearing & 114833 & $2.52 \%$ & $2,996,307$ & $2.50 \%$ & 34082 & $2.17 \%$ & $1,778,104$ & $2.46 \%$ \\
\hline Vision & 138,871 & $3.04 \%$ & $3,604,224$ & $3.01 \%$ & 34,390 & $2.19 \%$ & $1,791,838$ & $2.48 \%$ \\
\hline
\end{tabular}

${ }^{1}$ Person Inflation Ratio $=$ (weighted count $\div$ weighted total population $) ;{ }^{2}$ Unweighted Mexican-Latinos participants via MAIL; ${ }^{3}$ Weighted Mexican-Latinos participants via MAIL; ${ }^{4}$ Unweighted Mexican-Latinos participants via CATI/CAPI; ${ }^{5}$ Weighted Mexican-Latinos participants via CATI/CAPI; ${ }^{6}$ Unweighted Non-Latino-Whites participants via MAIL; ${ }^{7}$ Weighted Non-Latino-Whites participants via MAIL; ${ }^{8}$ Unweighted Non-Latino-Whites participants via CATI/CAPI; ${ }^{9}$ Weighted Non-Latino-Whites participants via CATI/CAPI 


\subsection{Non-Latino-Black vs. Non-Mexican-Latinos}

Table 6 indicates allocation rates are in general highest amongst Non-Latino-Blacks in all modes relative to Non-Mexican-Latinos/as and Vision remains the most allocation disability item. A small inflation of $0.22 \%$ on the allocation rate when population weights are applied is present in the Hearing and Vision for Non-Mexican-Latinos/as. Weighted allocation rates by race-ethnicity range from a low $1.53 \%$ to $7.08 \%$, they have a $\geq 92 \%$ level of completeness. PIR scores almost double from MAIL to CAPI/CATI.

Table 6. Unweighted and weighted allocations by race-ethnicity, mode, and disability item (universe $=53,507,740$ )

\begin{tabular}{|c|c|c|c|c|c|c|c|c|}
\hline \multirow[b]{4}{*}{ Self-Care } & \multicolumn{8}{|c|}{ Non-Latino-Black } \\
\hline & \multicolumn{4}{|c|}{ Mail $\left(P^{1}{ }^{1}=28.0\right)$} & \multicolumn{4}{|c|}{ CAPI/CATI $(P I R=54.5)$} \\
\hline & \multicolumn{2}{|c|}{$\mathrm{U}^{2} \mathrm{~N}=441,636$} & \multicolumn{2}{|c|}{$\mathrm{W}^{3} \mathrm{~N}=12,366,536$} & \multicolumn{2}{|c|}{$\mathrm{U}^{4} \mathrm{~N}=434,771$} & \multicolumn{2}{|c|}{$\mathrm{W}^{5} \mathrm{~N}=23,708,482$} \\
\hline & 31,221 & $7.07 \%$ & 867,559 & $7.02 \%$ & 11,085 & $2.55 \%$ & 568,565 & $2.40 \%$ \\
\hline Independent & 27,138 & $6.14 \%$ & 757,808 & $6.13 \%$ & 8,928 & $2.05 \%$ & 464,185 & $1.96 \%$ \\
\hline Ambulatory & 31,471 & $7.13 \%$ & 873,742 & $7.07 \%$ & 11,071 & $2.55 \%$ & 567,076 & $2.39 \%$ \\
\hline Cognitive & 30,968 & $7.01 \%$ & 859,382 & $6.95 \%$ & 11,368 & $2.61 \%$ & 580,820 & $2.45 \%$ \\
\hline Hearing & 28,194 & $6.38 \%$ & 784,544 & $6.34 \%$ & 11,637 & $2.68 \%$ & 594,801 & $2.51 \%$ \\
\hline \multirow[t]{4}{*}{ Vision } & 31,606 & $7.16 \%$ & 875,970 & $7.08 \%$ & 11,719 & $2.70 \%$ & 599,063 & $2.53 \%$ \\
\hline & \multicolumn{8}{|c|}{ Non-Mexican-Latinos/as } \\
\hline & \multicolumn{4}{|c|}{ Mail (PIR=28.3) } & \multicolumn{4}{|c|}{ CATI/CAPI $(P I R=56.3)$} \\
\hline & \multicolumn{2}{|c|}{$\mathrm{U}^{6} \mathrm{~N}=214,729$} & \multicolumn{2}{|c|}{$\mathrm{W}^{7} \mathrm{~N}=6,072,586$} & \multicolumn{2}{|c|}{$\mathrm{U}^{8} \mathrm{~N}=201,621$} & \multicolumn{2}{|c|}{$\mathrm{W}^{9} \mathrm{~N}=11,360,136$} \\
\hline Self-Care & 13,489 & $6.28 \%$ & 384,971 & $6.34 \%$ & 3,998 & $1.98 \%$ & 203,984 & $1.80 \%$ \\
\hline Independent & 11,061 & $5.15 \%$ & 319,369 & $5.26 \%$ & 3,314 & $1.64 \%$ & 173,874 & $1.53 \%$ \\
\hline Ambulatory & 13,503 & $6.29 \%$ & 385,296 & $6.34 \%$ & 3,986 & $1.98 \%$ & 203,562 & $1.79 \%$ \\
\hline Cognitive & 13,389 & $6.24 \%$ & 381,576 & $6.28 \%$ & 4,091 & $2.03 \%$ & 207,727 & $1.83 \%$ \\
\hline Hearing & 12,534 & $5.84 \%$ & 356,471 & $5.87 \%$ & 4,288 & $2.13 \%$ & 216,529 & $1.91 \%$ \\
\hline Vision & 13,252 & $6.17 \%$ & 376,447 & $6.20 \%$ & 4,305 & $2.14 \%$ & 217,763 & $1.92 \%$ \\
\hline
\end{tabular}

\footnotetext{
${ }^{1}$ Person Inflation Ratio $=$ (weighted count $\div$ weighted total population); ${ }^{2}$ Unweighted Non-Latino-Black participants via MAIL; ${ }^{3}$ Weighted Non-Latino-Black participants via MAIL; ${ }^{4}$ Unweighted Non-Latino-Black participants via CATI/CAPI; ${ }^{5}$ Weighted Non-Latino-Black participants via CATI/CAPI; ${ }^{6}$ Unweighted Non-Mexican-Latinos participants via MAIL; ${ }^{7}$ Weighted Non-Mexican-Latinos participants via MAIL; 8 Unweighted Non-Mexican-Latinos participants via CATI/CAPI; ${ }^{9}$ Weighted Non-Mexican-Latinos participants via CATI/CAPI
}

\section{Discussion}

The study is limited in that it does not account for variation in allocation rates by geography. It also omits a discussion on the fact that in theory, only one person participates with the 
survey. This means that the characteristics for more than three-fourths of all individuals are derived via "proxy report." Stratifying the present results by 'self' and 'proxy' report would have doubled the number of tables.

Because of space limitations, the project excludes the production of tables showing the confidence intervals around estimates-as presenting them would have significantly increased the number of tables and length of discussion. Notwithstanding these issues, the investigation is informative in that it clearly shows that a high level ( $\geq 92 \%)$ of within-person item-completeness (WPIC) for disability items is found in the microdata and that: vision is the most allocated disability item; allocations were most prevalent in MAIL; Person Inflation Ratios (PIRs) are lower in MAIL than in CAPI/CATI mode; and distortion between weighted and unweighted allocation rate was most frequently found with the independent, vision, and hearing disability items.

With regards to demographics, qualitative comparisons indicate: females had slight lower allocations rates; those with no college had higher rates of allocation in MAIL but only a slight increase in CAPI/CATI; Spanish only household in general had higher rates of allocation only in MAIL mode; married-couple family households had higher rates of allocation in CAPI/CATI than non-married-couple family households; allocation rates are highest amongst Mexican-Latinos/as than for Non-Latino-Whites only in MAIL mode; and allocation rates are in general highest amongst Non-Latino-Blacks in all modes relative to Non-Mexican-Latinos/as. The findings suggest higher levels of uncertainty in disability estimates could be found in data collected via MAIL mode.

Future work should produce disability estimates for these demographic variables by mode and their confidence intervals. Even more stark discoveries may be found along non-disability variables like personal income or educational attainment. The multidimensional complexity of allocations should be investigated through a more three-dimensional prism that considers geographical location as an important component of estimate variability. This is possible because observations in ACS PUMS files can be geographically referenced to Public Use Microdata Areas (PUMAs).

The US Census Bureau should consider providing the public with more detailed flag variables to identify when an edit refers to an assignment or allocation. They should also consider providing a variable that allows public data users the ability to disentangle the CAPI and CATI mode. Even more demanding is the suggestion that the US Census Bureau should consider providing the public with flag variables that can allow the identification of 'modified from missing' and 'modified from illogical' separately.

Beyond the esoteric issues raised in this technical manuscript is the fact that many of us have or will experience some degree of disablement during our lifetime. Understanding disability through a medical lens (e.g., capacity limitation) may obscure the fact that transitional or permanent impairment is partially produced by the fact that the social and physical environment is primarily built for able-body-people. Efforts should continue to build the operationalization of "disability" and methods for ascertaining its presence via force-choice-format survey questions. 


\section{Macrothink}

Issues in Social Science ISSN 2329-521X 2015, Vol. 3, No. 1

The current project hopes to inform on how disability data has the ability to inform social policy aimed at distributing resources to help equalize environmental inequality between the able and differently-able. This research contributes to the literature dealing with the estimation of disability prevalence and survey methodology by showing how within-person response mechanism may operate in data processing protocols to influence the quality of disability estimates. Because estimating the "true" prevalence of disability in the US population may help adequately fund the public health needs of those differently-abled, public health researchers should continue to explore how to cooperate with federal agencies to improve the estimation of disability and socially meaningful interpretation of estimates.

\section{References}

Alexander, C. H. (2002, July). A discussion of the quality of estimates from the American Community Survey for small population groups. In Draft presented at the Census Advisory Committee Meetings, September.

Battaglia, M. P., Izrael, D., Hoaglin, D. C., \& Frankel, M. R. (2004). Tips and tricks for raking survey data (aka sample balancing). Abt Associates (pp. 4740-4744).

Bishop, Yvonne M. M., Fienberg, Stephen E., \& Holland, Paul W. (1975). Discrete Multivariate Analysis:Theory and Practice. Cambridge, MA: MIT Press.

Brault, M. W. (2012). Americans with disabilities: 2010. US Department of Commerce. Economics and Statistics Administration. US Census Bureau.

Brault, M., Stern, S., \& Raglin, D. (2007). Evaluation report covering disability. 2006 American Community Survey Content Test Report (Part 4).

Brick, J. M., Montaquila, J., \& Roth, S. (2003). Identifying Problems with Raking Estimators. 2003 ASA Proceedings [CD-ROM] (pp. 710-717). Alexandria, VA: American Statistical Association.

Brick, J. M. (2013). Unit Nonresponse and Weighting Adjustments: A Critical Review. Journal of Official Statistics, 29(3), 329-353.

Couper, M. P. (2011). The future of modes of data collection. Public opinion quarterly, 75(5), 889-908.

Chen, J., \& Shao, J. (2000). Nearest neighbor imputation for survey data. Journal of Official Statistics-Stockholm, 16(2), 113-132.

Kim, J. K., \& Fuller, W. A. (2004). Inference procedures for hot deck imputation. Biometrika, 91, 559-578.

Kim, J. K., Fuller, W. A., \& Bell, W. R. (2008). Variance Estimation for Nearest Neighbor Imputation for US Census Long Form Data. Statistics, 13.

Kim, J. K., Fuller, W. A., \& Bell, W. R. (2011). Variance estimation for enarest neighbor imputaion for US Census long form data. The Annals of Applied Statistics, 5, 824-842. 


\section{Macrothink

Carrasco, L. (2003). The American Community Survey (ACS) en Español: Using cognitive interviews to test the functional equivalency of questionnaire translations (Study Series). Survey Methodology, 17.

de Leeuw, E. D. (1993). Mode effects in survey research: a comparison of mail, telephone, and face to face surveys. Bulletin de Méthodologie Sociologique, 41(1), 3-19.

de Leeuw, E. D. (2005). To mix or not to mix data collection modes in surveys. Journal of official statistics, 21(5), 233-255.

de Leeuw, E. D., Hox, J. J., \& Dillman, D. A. (2008). Mixed-mode surveys: When and why. International handbook of survey methodology, 299-316.

de Leeuw, W., de Leeuw, E. D., \& van der Zouwen, J. (1999). Methodological issues in survey research: a historical overview. Bulletin de Methologie Sociologique, 64, 25-48.

Deming, W. E. (1944). On errors in surveys. American Sociological Review, 9,359-369.

Deming, W. E. (1943), Statistical Adjustment of Data. New York: Wiley.

Deville, J. C., Särndal, C. E., \& Sautory, O. (1993). Generalized raking procedures in survey sampling. Journal of the American Statistical Association, 88(423), 1013-1020.

Deville, J. C., \& Särndal, C. E. (1992). Calibration estimators in survey sampling. Journal of the American Statistical Association, 87(418), 376-382.

Eaton, D. K., Brener, N. D., Kann, L., Denniston, M. M., McManus, T., Kyle, T. M., ... \& Ross, J. G. (2010). Comparison of paper-and-pencil versus Web administration of the Youth Risk Behavior Survey (YRBS): risk behavior prevalence estimates. Evaluation review, 34(2), 137-153.

Fuchs, M., Couper, M., \& Hansen, S. E. (2000). Technology effects: Do CAPI or PAPI interviews take longer? Journal of Official Statistics-Stockholm, 16(3), 273-286.

Hochstim, J. R. (1967). A critical comparison of three strategies of collecting data from households. Journal of the American Statistical Association, 62, 976-989.

Joshipura, M. (2008). 2005 ACS Respondent Characteristics Evaluation. US Census Bureau.

Joshipura, M. (2010, August). Evaluating the effects of a multilingual brochure in the American Community Survey. In 65th Annual American Association for Public Opinion Research Conference, Chicago, May (pp. 13-16).

Kish, L. (1981). Using cumulated rolling samples to integrate Census and survey operations of the Census Bureau: an analysis, review, and response. Washington, DC: U.S. government Printing Office.

Little, R. J. A. (1993). Post-stratification: A modeler's perspective. Journal of the American Statistical Association, 88, 1001-1012. 


\section{Macrothink}

Issues in Social Science ISSN 2329-521X 2015, Vol. 3, No. 1

Lumley, T. (2004). Analysis of complex survey samples. Journal of Statistical Software, 9(1), $1-19$.

McGovern, P. D., \& Griffin, D. H. (2003, August). Quality Assessment of Data Collected from Non-English Speaking Households in the American Community Survey. Joint Statistical Meetings, San Francisco, August (Vol. 3).

McGovern, P. D. (2004). A quality assessment of data collected in the American Community Survey (ACS) from households with low English proficiency. Survey Methodology, 1.

Reamer A. D. (2010). Surveying for Dollars: The Role of the American Community Survey in the geographic distribution of federal funds. Metropolitan Policy Program at Brookings, Washington D.C.

Salvo, J. J., \& Lobo, A. P. (2002). The American Community Survey: Quality of response by mode of data collection in the Bronx test site. In Joint Statistical Meetings of the American Statistical Association. New York, NY.

Shao, J. (2009). Nonparametric variance estimation for nearest neighbor imputation. Journal of Official Statistics, 25, 55-62.

Shao, J. and Wang, H. (2008). Confidence intervals based on survey data with nearest neighbor imputation. Statist Sinica, 18, 281-297.

Siordia C. (2013). Methodological Note: Allocations with Health Insurance Items in the American Community Survey. Journal of Frailty and Aging, 6(2), 149-153. http://dx.doi.org/ 10.1016/j.dhjo.2012.11.007

Siordia C., \& Young, R. L. (2013). Methodological Note: Allocation of Disability Items in the American Community Survey. Disability and Health Journal. http://dx.doi.org/ 10.1016/j.dhjo.2012.11.007

Siordia C, \& Le, V. D. (2013). Precision of Disability Estimates for Southeast Asians in American Community Survey 2008-2010 Microdata. Central Asian Journal of Global Health, 1(2). http://dx.doi.org/10.5195/cajgh.2012.2166-7403

Torrieri, N. K. (2007). America is Changing, and So is the Census. The American Statistician, 61(1).

Tourangeau, R. (2004). Survey research and societal change. Annu. Rev. Psychol., 55, 775-801. 